\title{
Carbamazepine, Sodium Valproate and Levetiracetam Modulate Wnt Inhibitors in Indian Women with Epilepsy
}

\author{
Bushra Parveen ${ }^{\prime}$, Manjari Tripathi', Divya Vohora ${ }^{l}$ \\ Pharmaceutical Medicine, Department of Pharmacology, \\ School of Pharmaceutical Education and Research, Jamia Hamdard, New Delhi ${ }^{1}$ \\ Department of Neurology, Neurosciences Centre, \\ All India Institute of Medical Sciences, New Delhi .
}

\begin{abstract}
Background: Antiepileptic drug (AED) therapy has been claimed to deteriorate bone health. Majority of the research was inclined towards vitamin-D deficiency as the patho-mechanism. However, after the role of $W n t$ in bone metabolism was discovered, it has paved way for investigating the role of $W n t$ inhibitors in mediating effects on bone accrual. Recently, we have reported the modulation of two Wnt inhibitors, sclerostin and dickkopf-1 (DKK-1), following AED therapy in Indian women with epilepsy, however, the subgroup analysis for individual drug is elucidated in this report.
\end{abstract}

Methods: Individual analysis for our earlier cross-sectional study on three AEDs, carbamazepine (CBZ), sodium valproate (SVP) and levetiracetam (LTM), on sclerostin and dickkopf-1, and their correlation with receptor activator of nuclear factor kappaB ligand (RANKL) and serum 25-hydroxy vitamin D (25OHD) was assessed in Indian women with epilepsy.

Results: We observed enhanced sclerostin and 25OHD levels with all three AEDs while serum RANKL was higher with SVP and LTM only. Further, serum DKK-1 levels were lowered with CBZ and LTM. Sclerostin showed a positive correlation with RANKL in CBZ group, while DKK-1 presented no such relationship.

Conclusion: As sclerostin is more specific than DKK-1, we may conclude that these drugs may compromise bone health through disturbance in Wnt signaling mechanisms.

Keywords: sclerostin, DKK-1, RANKL, 25OHD, sodium valproate, carbamazepine, levetiracetam.

\section{Introduction}

Wht signaling plays an important role in development and maintenance of many organs and tissues, including bone. Wnt/ $\beta$-catenin or canonical pathway appears to be particularly important for bone biology (1). Wnt signaling is tightly regulated by members of several families of secreted antagonists including sclerostin and dickkopf-1 (DKK-1) (2). Previous reports have identified vitamin-D inactivation as a major cause of antiepileptic drugs (AEDs) induced metabolic bone diseases (3). However, it is required to explore newer mechanisms that may aid in understanding the pathophysiology of AED induced bony consequences better. The

Correspondence: Prof. Divya Vohora, Department of Pharmacology, School of Pharmaceutical Education and Research, In-charge, Pharmaceutical Medicine, Jamia Hamdard (Deemed to be University), New Delhi-110062.Ph:91-11-26059688, Extn.5657.Email: divyavohora@hotmail.com, dvohra@jamiahamdard.ac.in. 
objective of our study was primarily to perform sub-group analysis on our previously published report on the modulation of Wnt inhibitors and receptor activator of nuclear factor kappaB ligand (RANKL) following AED monotherapy. It is well-known that DKK-1 and sclerostin, secreted by osteocytes, block the effect of Wnt on osteoblasts thereby reducing bone formation. Sclerostin has been reported as a strong and independent risk factor for osteoporosis related fractures among postmenopausal women (4). On the other hand, RANK/RANKL pathway plays a critical role in bone remodeling and higher RANKL levels corresponds to increased bone resorption. Several studies provide evidence of association of these markers with bony consequences (5-7). This study aims to look for evidence of any association between $W n t$ inhibitors and three AEDs.

\section{Method}

The study includes sub-group analysis of data of a cross-sectional analytic study conducted at Neuroscience Centre, AIIMS, New Delhi and has been published recently (8). Parveen et al included 32 adult women with epilepsy treated with sodium valproate (SVP), carbamazepine (CBZ) or levetiracetam (LTM) as monotherapy for at least a year. All women gave their consent in accordance with declaration of Helsinki. Serum was employed for the analysis of $W n t$ inhibitors (Sclerostin and DKK-1) and RANKL using human specific ELISA kit from Sincere Biotech (Beijing, People's Republic of China) and 25OHD procured from Siemens (Mumbai, India).

Data contained both continuous and categorical variables. Therefore, mean $\pm \mathrm{SD}$ or median with interquartile range (IQR) were used for continuous data whereas frequency with proportions were used for categorical data for their presentation. Normality of the continuous variables was tested by the KolmogorovSmirnov and Shapiro-Wilk tests. Analysis of variance (ANOVA) test was used between treatment groups and post-hoc analysis was done by using Tukey's honestly significant difference test. ANOVA test and post-hoc Dunnett t-test was used for comparison between individual drug and control. For this comparison, RANKL was converted to log form as data was not normally distributed. The Spearman rank correlation was used to assess the correlation between the variables. The two-sided p-value less than 0.05 was considered as statistical significant. The statistical software IBM SPSS Version 22.0 (Chicago, USA) was used for entire analysis.

\section{Results}

\section{Demographics and Clinical Characteristics}

Thirty-two women with epilepsy were included in the study. Based on the drug treatment, patients were stratified into individual drug treatment groups comprising CBZ $(n=08)$, SVP $(n=10)$ and LTM $(n=14)$. There was no statistical difference in age and duration of treatment among groups receiving various AEDs (Table 1). Patients receiving LTM achieved highest seizure control $(78.6 \%)$ at mean total daily dose of $1250 \pm 353.55 \mathrm{mg} / \mathrm{kg}$ as compared to CBZ $(62.5 \%)$ and SVP $(50 \%)$.

\section{Biochemical and Correlation Analysis}

Sclerostin levels were higher in patients undergoing treatment with all three AEDs while serum RANKL levels were significantly higher in SVP group and LTM but not with CBZ. 25OHD levels were lowered in patients undergoing treatment with all three AEDs studied in individual AED analysis. Serum DKK-1 levels were significantly lowered with CBZ and LTM but not with SVP (Table 2). The mean 25OHD level was significantly more among the serum levels of patients treated with CBZ (mean difference, 4.11) and LTM (mean difference, 4.11) in comparison to SVP. The mean sclerostin was significantly more in LTM in comparison to CBZ (mean difference, -3.60) while mean DKK-1 was significantly less in CBZ compared to SVP (mean difference, 
Table 1: Demographical and clinical characteristics of women with epilepsy included in the clinical study

\begin{tabular}{|c|c|c|c|}
\hline \multirow[b]{2}{*}{ Characteristics } & \multicolumn{3}{|c|}{ Women with epilepsy on AEDs } \\
\hline & $\begin{array}{l}\text { CBZ } \\
\text { n=08 }\end{array}$ & $\begin{array}{l}\text { SVP } \\
n=10\end{array}$ & $\begin{array}{c}\text { LTM } \\
n=14\end{array}$ \\
\hline $\begin{array}{l}\text { Age } \\
\text { (mean } \pm \mathrm{SD}, \text { years) } \\
\text { (range) }\end{array}$ & $\begin{array}{c}26 \pm 6.39 \\
(20-36)\end{array}$ & $\begin{array}{c}27.7 \pm 6.13 \\
(20-39)\end{array}$ & $\begin{array}{c}24.64 \pm 5.03 \\
(20-38)\end{array}$ \\
\hline $\begin{array}{l}\text { Type of seizure } \\
\text { Generalized tonic clonic } \\
\text { Partial } \\
\text { Febrile } \\
\text { Juvenile myoclonic } \\
\text { Absence } \\
\text { Partial seizure with } \\
\text { secondary generalization }\end{array}$ & $\begin{array}{l}5 \\
3 \\
0 \\
0 \\
0 \\
0\end{array}$ & $\begin{array}{l}5 \\
3 \\
0 \\
0 \\
1 \\
1\end{array}$ & $\begin{array}{l}9 \\
3 \\
1 \\
1 \\
0 \\
0\end{array}$ \\
\hline Family history & 0 & 0 & 1 \\
\hline $\begin{array}{l}\text { Seizure -free period } \\
\text { (mean } \pm \mathrm{SD}, \text { months) } \\
\text { (range) }\end{array}$ & $\begin{array}{c}29.12 \pm \\
29.33 \\
(3-72)\end{array}$ & $\begin{array}{c}23.5 \pm 39.41 \\
(1-133)\end{array}$ & $\begin{array}{c}23.5 \pm 15.65 \\
(1-41)\end{array}$ \\
\hline Total daily dose of AEDs (mg/day) & 400 & 500 & $1250 \pm 353.55$ \\
\hline $\begin{array}{l}\text { Duration of } t \text { reatment } \\
\text { (mean } \pm \mathrm{SD} \text {, months) } \\
\text { (range) }\end{array}$ & $\begin{array}{c}71.5 \pm 51.07 \\
(24-190)\end{array}$ & $\begin{array}{c}48.6 \pm 40.57 \\
\quad(13-132)\end{array}$ & $\begin{array}{c}40.07 \pm 22.24 \\
(12-72)\end{array}$ \\
\hline $\begin{array}{l}\text { Degree of control on AEDs } \mathrm{n}(\%) \\
\text { Controlled } \\
\text { (seizure free for > } 1 \text { yr or more) } \\
\text { Uncontrolled }\end{array}$ & $\begin{array}{l}5(62.5 \%) \\
3(37.5 \%)\end{array}$ & $\begin{array}{l}5(50 \%) \\
5(50 \%)\end{array}$ & $\begin{array}{l}11(78.6 \%) \\
3(21.4 \%)\end{array}$ \\
\hline
\end{tabular}

Note: The results are expressed numbers, $\%$ or Mean $\pm \mathrm{SD}$.

AEDs- Anti-epileptic drugs; CBZ- Carbamazepine; SVP- Sodium valproate; LTM- Levetiracetam.

-147.2). Sclerostin had a significant positive correlation with RANKL in CBZ group (Fig. 1), while DKK-1 showed no such relationship with any of the AEDs studied.

\section{Discussion}

There is increasing evidence that AED therapy is associated with alteration in bone metabolism and mineralization suggesting bone loss. However, there is no generalized mechanism responsible for such consequences. In our previous study, alteration in Wnt inhibitors was reported following AED therapy in Wistar rats (9) and in Indian women with epilepsy (8). The present study analyzed the effect of individual drugs viz SVP, CBZ and LTM on modulation of Wnt inhibitors in persons with epilepsy. Similar to previous study by Gifre et al (10) evaluating the effect of glucocorticoids on modulation of Wnt inhibitors, the present study also reported differential effects with the type of 
Table 2: Bone turnover markers (RANKL and 25(OHD) and $W n t$ antagonists (Sclerostin and DKK-1) in persons with epilepsy on AED monotherapy in comparison to age-matched healthy controls

\begin{tabular}{|c|c|c|c|}
\hline Bone turnover markers & CBZ & SVP & LTM \\
\hline RANKL $\left(\mathbf{p g m L}^{-1}\right)$ & $371.37(347.11)$ & $688.81(469.78)^{\# \# \#}$ & $482.94(475.16)^{\# \# \#}$ \\
\hline 25OHD $\left(\mathbf{n g m L}^{-1}\right)$ & $26.40(4.45)^{\# \#}$ & $22.29(2.89)^{\# \# ~ a ~}$ & $26.40(4.00)^{\# \# \#}$ \\
\hline Sclerostin $\left(\mathbf{n g m L}^{-1}\right)$ & $15.11(2.09)^{\# \#}$ & $16.79(2.62)^{\# \# \#}$ & $18.71(4.90)^{\# \# \mathrm{~b}}$ \\
\hline DKK-1 $\left.\mathbf{( p g m L}^{-1}\right)$ & $749.88(57.46)^{\# \# \text { c }}$ & $897.14(126.30)$ & $800.65(690.96)^{\# \# \#}$ \\
\hline
\end{tabular}

RANKL- receptor activator of nuclear factor kappaB ligand; DKK-1- Dickkopf-1; 25OHD- 25hydroxy vitamin D; CBZ- Carbamazepine; SVP- Sodium valproate; LTM- Levetiracetam.

Note: Data are expressed as mean (SD); RANKL was converted to log RANKL for comparison between CBZ, SVP and LTM vs Control. Values of control group for RANKL, 25OHD, sclerostin and DKK-1 has been taken from Parveen et al 2018 for comparison.

\#\#\#p < 0.001; CBZ, SVP, LTM vs control (ANOVA followed by post-hoc Dunnett t-test) ${ }^{\mathrm{a} S V P}$ vs LTM ( $\mathrm{p}=0.037$, ANOVA followed by post-hoc Tukey HSD Multiple Comparisons Tests) ${ }^{b}$ LTM vs CBZ ( $p=0.007$, ANOVA followed by post-hoc Tukey HSD Multiple Comparisons Tests) ${ }^{c} \mathrm{CBZ}$ vs SVP ( $p=0.019$, ANOVA followed by post-hoc Tukey HSD Multiple Comparisons Tests).

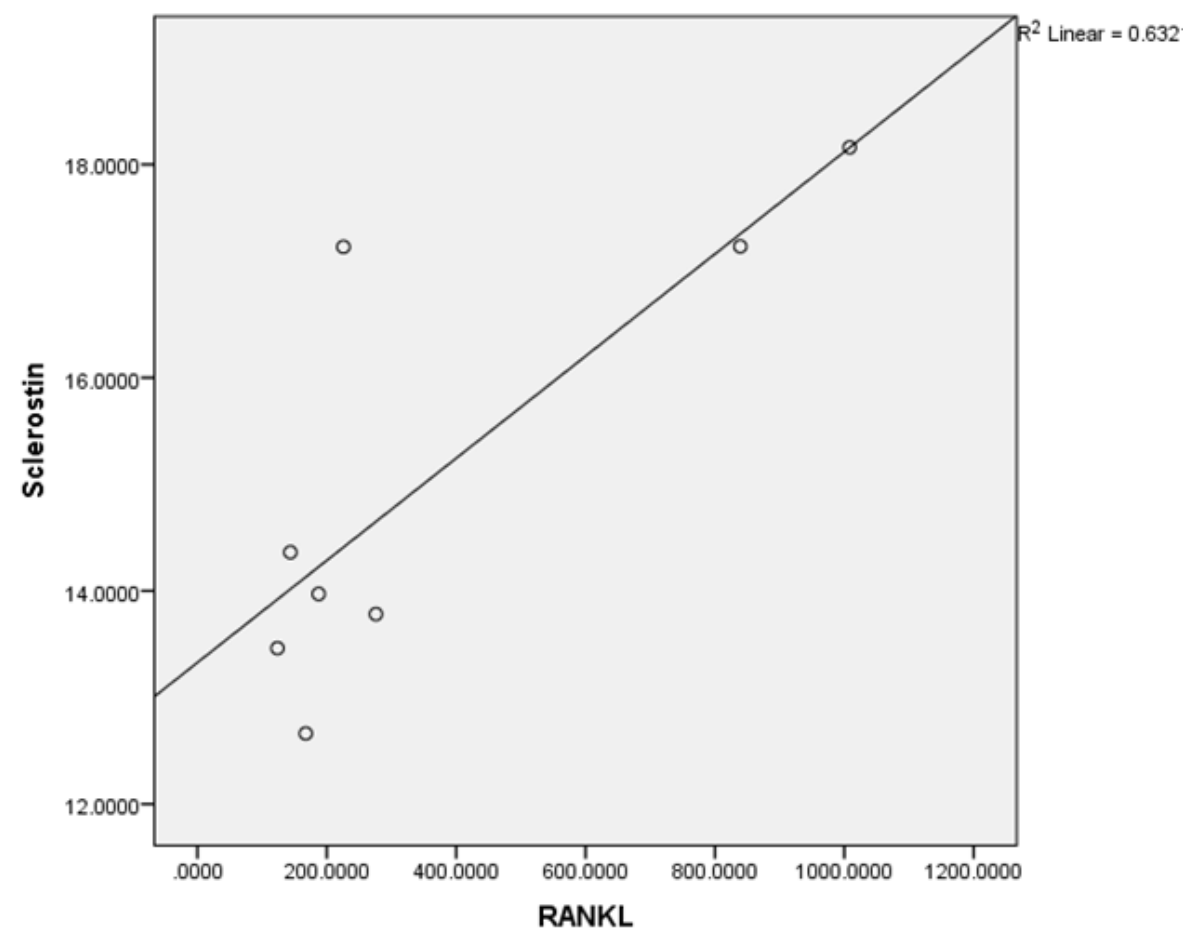

Fig. 1: Positive correlation between Sclerostin and RANKL in treatment with CBZ. Correlation is significant at the 0.01 level (2-tailed). 
Wnt inhibitor evaluated. While there were higher sclerostin levels with all three AEDs, DKK-1 levels were lowered with CBZ and LTM. Highest sclerostin levels were reported with LTM while lowest DKK-1 levels with CBZ. However, in our study, the observed lower DKK1 levels following AED therapy in persons with epilepsy was not significant with SVP in individual AED analysis. DKK-1 plays a critical role during skeletal development but it is not highly expressed in adult bone unless activated by an insult (11). Sclerostin expression is limited primarily to the skeleton whereas expression of DKK-1 is more widespread (12).

The higher serum RANKL observed in our study following SVP is in agreement with preclinical study demonstrating increased RANKL levels in rats treated with valproate (13). RANKL activates and/or induces various transcription factors including nuclear factor kappa-light-chain-enhancer of activated B cells (NF- $\mathrm{KB}$ ), c-Fos and nuclear factor of activated Tcells c1 (NFATc1), which act as positive modulators of osteoclast differentiation (14). RANKL increases NFATc1 protein levels by post-translational modification. RANKL stimulates NFATc1 acetylation via histone acetyltransferases (HATs). RANKL-mediated NFATc1 acetylation is increased by the histone deacetylase (HDAC) inhibitors (15). Various AEDs such as valproic acid, CBZ, oxcarbazepine, lamotrigine and levetiracetam are known to exert histone deacetylase inhibitory (HDACi) properties (16). Hence, increased RANKL levels by SVP and LTM could also be related to its HDAC inhibiting property (17). Though, CBZ didn't exert significant rise in serum RANKL levels but showed an increasing trend. The role of $W n t$ inhibitors and RANKL in bony consequences following AED therapy is proven in our previous report where sclerostin was positively correlated with RANKL, while no such effects were observed with DKK-1 (8). Now the individual analysis of drugs showed that the positive correlation observed between sclerostin and RANKL is due to CBZ. 25OHD was lowered with all three AEDs compared to control, however, the serum levels were not in the deficient range. Interestingly, our study showed that decrease in 25OHD was highest in SVP group as compared to CBZ and LTM treatment group. However, our study had limitation as the baseline data for 250HD was not available and we compared the results with healthy controls.

To conclude, AEDs may compromise bone health through alterations in Wnt pathway via enhanced sclerostin levels which may or may not be related to RANKL depending upon the type of AED investigated.

\section{List of Abbreviations}

AEDs- Antiepileptic drugs

CBZ- Carbamazepine

SVP- Sodium valproate

LTM- Levetiracetam

RANKL- Receptor activator of nuclear factor kappaB ligand

DKK-1- Dickkopf 1

NF-кB- Nuclear factor kappa-light-chainenhancer of activated B cells

HDAC- Histone deacetylase

NFATc1 - Nuclear factor of activated T-cells c1

CPCSEA- Committee for the Purpose of Control and Supervision of Experiments on Animals

ANOVA- Analysis of variance

\section{Acknowledgment}

We are grateful to the University Grants Commission- Special Assistance Programme (UGC-SAP) for financial assistance. BP is thankful to Department of Science and Technology, New Delhi, for providing DSTINSPIRE Fellowship, Ref No. ST/INSPIRE FELLOWSHIP/2014/IF140811. 


\section{Author Contributions Statement}

$\mathrm{BP}$ is responsible for enrolling patients, biochemical and statistical analysis and writing draft of the manuscript; MT is responsible for screening patients and editing the manuscript; DV is responsible for conception of study, scientific support and final editing of manuscript.

\section{Conflict of Interest}

The authors declare no conflict of interest.

\section{References}

1. Krishnan V, Bryant HU, MacDougald OA (2006). Regulation of bone mass by Wnt signaling. JClin Invest 116: 1202-1209.

2. Kim JH, Liu X, Wang J, et al (2013). Wnt signaling in bone formation and its therapeutic potential for bone diseases. Ther Adv Musculoskelet Dis 5: 13-31.

3. Albaghdadi O, Alhalabi MS, Alourfi Z, Lama A (2016). Bone health and vitamin D status in young epilepsy patients on valproate monotherapy. Clin Neurol Neurosurg 146: 52-56.

4. Ardawi MS, Rouzi AA, Al-Sibiani SA, AlSenani NS, Qari MH, Mousa SA (2012). High serum sclerostin predicts the occurrence of osteoporotic fractures in postmenopausal women: The Center of Excellence for Osteoporosis Research Study. J Bone Miner Res 27: 2592-2602.

5. Uemura H, Yasui $\mathrm{T}$, Miyatani $\mathrm{Y}$, et al (2008). Circulating profiles of osteoprotegerin and soluble receptor activator of nuclear factor kappaB ligand in post-menopausal women. J Endocrinol Invest 3: 163-168.

6. Oh KW, Rhee EJ, Lee WY, et al (2005). Circulating osteoprotegerin and receptor activator of NF-kappaB ligand system are associated with bone metabolism in middle-aged males. Clin Endocrinol (Oxf) 62: 92-98.

7. $\mathrm{Xu} \mathrm{XJ}$, Shen L, Yang YP, et al (2013). Serum $\beta$-Catenin levels associated with the ratio of RANKL/OPG in patients with postmenopausal osteoporosis. Intl $J$ Endocrinol 2013: 534352.

8. Parveen B, Tripathi M, Vohora D (2018). A cross-sectional study to assess the modulation of $W n t$ Inhibitors following anti-epileptic drug therapy and their correlation with vitamin $\mathrm{D}$ and receptor activator of nuclear factor $\kappa \mathrm{B}$ ligand in Indian women with epilepsy. Basic Clin Pharmacol Toxicol 123(3): 271-276.

9. Parveen B, Tiwari AK, Jain $\mathrm{M}$, et al (2018). The anti-epileptic drugs valproate, carbamazepine and levetiracetam cause bone loss and modulate $W n t$ inhibitors in normal and ovariectomised rats. Bone 113: 57-67.

10. Gifre L, Ruiz-Gaspa S, Monegal A, et al (2013). Effect of glucocorticoid treatment on Wnt signalling antagonists (sclerostin and DKK-1) and their relationship with bone turnover. Bone 57: 272-276.

11. Li X, Grisanti M, Fan W, et al (2011). Dickkopf-1 regulates bone formation in young growing rodents and upon traumatic injury. J Bone Miner Res 26: 2610-2621.

12. Ke HZ, Richards WG, Li X, Ominsky MS (2012). Sclerostin and dickkopf-1 as therapeutic targets in bone diseases. Endocrine Rev 33: 747-783.

13. Elwakkad AS, El Elshamy KA, Sibaii H (2008). Fish liver oil and propolis as protective natural products against the effect of the anti-epileptic drug valproate on immunological markers of bone formation in rats. Epilepsy Res 80: 47-56. 
14. Teitelbaum SL, Ross FP (2003). Genetic regulation of osteoclast development and function. Nat Rev Genet 4: 638-649.

15. Kim JH, Kim K, Youn BU, et al (2011). RANKL induces NFATc1 acetylation and stability via histone acetyl transferases during osteoclast differentiation. Biochem J436: 253-262.

16. Stettner M, Krämer G, Strauss A, et al (2012). Long-term antiepileptic treatment with histone deacetylase inhibitors may reduce the risk of prostate cancer. Eur $J$ Cancer Prev 21: 55-64.
17. Göttlicher M, Minucci S, Zhu $\mathrm{P}$, et al (2001). Valproic acid defines a novel class of HDAC inhibitors inducing differentiation of transformed cells. EMBOJ 20: 6969-6978. 